\title{
Renormalization in Reparameterization Invariance
}

\author{
Yu-Qi Chen \\ Institute of Theoretical Physics, Academia Sinica, Beijing 100080, P.R. of China
}

\begin{abstract}
The renormalization issue of the reparameterization invariance in heavy quark effective theory and NRQCD is investigated. I argue that the renormalization of the transformation of the heavy quark field under the variation of the velocity parameter $V$ is attributed to the renormalization of the small component field in the proposed transformation. I show that the matching condition for determining the renormalized small component field can be obtained by imposing an infinitesimal transformation of $V$ on the relations between the Green's functions in QCD full theory and those in the effective theory. As an application, I determine the renormalized transformation to order $1 / m^{2}$ using the matching condition. The obtained result is in disagreement with that determined by indirect method.
\end{abstract}




\section{INTRODUCTION}

Heavy quark effective theory (HQET) [1] and nonrelativistic QCD (NRQCD) 2] are powerful tools in dealing with dynamics of heavy-light and heavy-heavy systems, respectively. In those systems, the off-shell momentum of the heavy quark is much smaller than its mass. The effective theories are designed to reproduce the results of the QCD full theory at the low energy scale in a simpler way by integrating out the effects at the energy scale of the heavy quark mass. In the past decade both effective theories and their applications have been intensively studied.

One interesting theoretical issue in those effective theories is the reparameterization invariance (RPI). It arises from the fact that the effective theory explicitly depends on the four velocity parameter $V$. In constructing the effective lagrangian, one needs to divide the heavy quark momentum $P$ into a large part and a small one as $P=m V+k$, where $m$ is the heavy quark mass and $k$ is a small residual momentum. one also needs to decompose the Dirac 4-fermion field as large and small two component fields in respect of $V$ and uses the large one to describe the heavy quark or antiquark. These procedures lead to the effective lagrangian being $V$-dependent. The choice of $V$ which satisfies $V^{2}=1$ is not unique. But the physical prediction should be unchanged against the variation of the velocity parameter $V$. This is the RPI. It is required by the consistence of the effective theory and also conducts interesting applications. It was first proposed in HQET. However, the same invariance also holds in NRQCD effective theory.

To implement RPI in the effective theory, it is essential to find out an appropriate transformation of the heavy quark field under the variation of $V$, which is found to be quite nontrivial. It was first studied by Manohar and Luke[3] in HQET. They used the Lorentz boost of spinor field as the transformation of the heavy quark field from finite velocity $V \rightarrow V^{\prime}$. Their transformation suffers from operator ordering ambiguities when it is expanded to a higher order of $1 / m$ while Manohar[4] discussed its expansion at higher order. Chen [5] proposed an infinitesimal transformation of the heavy quark field under the velocity variation from $V \rightarrow V+\Delta V$. Chen's transformation keeps tree level effective lagrangian invariant to all orders of $1 / m$. Finkemeier, Georgi, and McIrvin[ [6] showed that to order $1 / m^{2}$ the effective lagrangian constrainted by Manohar and Luke's transformation and Chen's transformation may be related by a field redefinition. 
Chen's transformation can be expanded as inverse power series of the heavy quark mass. Each term contains the product of some covariant derivatives and the heavy quark field. Since this kind of expansion changes the ultraviolet behavior of the original transformation, beyond the next leading order, it turns out that it needs to be renormalized. The renormalization of it is different from that of the effective lagrangian in which each term is the bilinear function of the heavy quark fields. For that case, we have appropriate matching conditions to determine the renormalized transformation.

Kilian and Ohl[7] proposed a renormalized transformation. The form is exactly the same with Chen's transformation except that the covariant derivative $D^{\mu}$ in Chen's transformation is substituted by another operator which they called as the general covariant derivative. Sundrum [8] discussed this issue using the auxiliary field method and obtained a result similar with Kilian and Ohl's. The results presented in these papers are formal. They did not showed how to determine the general covariant derivative by some specific matching conditions. Actually, in literatures no specific calculation for determining the transformation has been done with this method. The only calculation to determine the renormalized transformation is given by Balzereit in an unpublished paper [9]. He first calculated the effective lagrangian to order $1 / \mathrm{m}^{3}$ at one loop level in the leading logarithmic approximation. By requiring the effective lagrangian invariant, he then determined the renormalized transformation to order $1 / m^{2}$ indirectly. There are some drawbacks in this kind of calculations. First, they are quite complicated since the determination of higher order effective lagrangian usually is hard work. Second, it makes the RPI less practical application. An interesting application of the RPI is that once we know the transformation, we can use it to constraint the higher order effective lagrangian and makes the calculations simpler [3, 5, 6]. Balzereit did it in an inverse order so RPI may not be used to constraint the effective lagrangian in his method.

In this paper, I study this issue in an alternative way, with emphasizing on determining the renormalized transformation using the matching conditions. It is essential in studying the renormalization issue of RPI. With it, one is able to determine the renormalized transformation to any desired order both in $\alpha_{s}$ and in $1 / m$ expansion.

To derive the matching condition, it is instructive to recall the renormalization procedure of the effective lagrangian. There are simpler relations between the Green's function in the unexpanded effective theory with nonlocal form. These relations ensure that the effective theory and the full QCD reproduce the same physical predictions. The expanded effective 
theory changes the ultraviolet behavior of the unexpanded effective theory. In order to reproduce the same result of the full QCD, those relations are required to be satisfied and one has to add some local operators in the effective lagrangian, i.e., renormalizing the effective lagrangian. Therefore, those relations between the Green's functions can be used as the matching conditions [1] to determine the effective lagrangian. On the other hand, there is no direct relations between the 1PI vertexes in the effective theory and those in the full QCD. Thus one may not obtain the matching conditions from the 1PI vertex.

As we will see later on, the renormalization of the transformation of the heavy quark field under the variation of the velocity parameter $V$ is attributed to the renormalization of the small component field in the proposed transformation. Therefore, if we can find some relations between certain correlation functions in the full QCD and those in the effective theory, which involve the small component field, it then can be used as the matching conditions to determine the renormalized transformation. It is found that imposing an infinitesimal transformation on both sides of the relations between the Green's functions in QCD full theory and those in the effective theory, new relations in which the small component field is involved on the effective theory side are obtained. These new relations are nothing but the matching conditions to determining the renormalized small component field. They provide a systematic way to determine the renormalized transformation. As a specific example, I use these matching conditions to determine the renormalized transformation to order $1 / \mathrm{m}^{2}$ at one-loop level. The obtained result is in disagreement with that obtained by Balzereit 9$]$. Since the same lagrangian (MRR lagrangian) are used, the disagreement between these two different results cannot be accounted by a field redefinition. I then show that the renormalized transformation determined by these matching conditions can be written in the form of Chen's transformation with the covariant derivative substituted by an operator which may be called as a general covariant derivative. Thus renormalized transformation determined by the matching conditions presented in this paper is consistent with Kilian and Ohl's result. [7]

The remainder of the paper is organized as follows. In section II, after a brief review on the tree level transformation, I argue that the renormalized transformation of the heavy quark field is attributed to the renormalization of the small component field. I then show that the matching condition for determining it can be obtained by imposing an infinitesimal transformation on the general relations between the Green's functions in QCD full theory and those in the effective theory. As an example, in section III, I determine the renormalized 
transformation to order $1 / \mathrm{m}^{2}$ by matching the two-point and the three-point functions. In section IV, I show that previous results can be more clearly understood in an alternative way to construct the effective lagrangian, where a four-component effective lagrangian constructed first, followed by its reduced into the effective lagrangian in a two-component field. I then show that the remormalized small component field determined by the matching conditions are consistent with Kilian and Ohl's result. [7]. Then I shown that the remormalized effective lagrangian is reparameterization invariant under the renormalized transformation. Section V contributes to the conclusion. Finally, in appendix A, I derive the general relation between the Green's functions using the generating functional method.

\section{RENORMALIZED TRANSFORMATION OF THE HEAVY QUARK FIELD}

In the heavy quark effective theory, the heavy quark is described by a two component field while in QCD full theory it is described by the Dirac four-component field. Thus, to construct the effective theory, one needs first to decompose the Dirac four-component field into two-component field. A simpler way to realize this decomposition introduced by Georgi 1] is:

$$
h_{V \pm}(x) \equiv \exp (i m V \cdot x) \mathrm{P}_{ \pm} \Psi(x)
$$

where

$$
\mathrm{P}_{ \pm} \equiv \frac{1 \pm V}{2}
$$

are the projection operators. The phase factor just removes the large part of the heavy quark momentum when it is written as $p=m V+k$. This definition of the field is used by most people 1] in literatures. Nevertheless, it is not unique. Different definitions lead to different forms of the effective lagrangian. However, they can be related to each other by field redefinition and produce the same physical predictions [6]. Overall in this paper, we use the definition of (11).

\section{A. the tree level transformation}

With the definition (11), the effective lagrangian reads [5, [13]:

$$
\mathcal{L}_{\mathrm{eff}}^{0}=\bar{h}_{V+}(x) i D \cdot V h_{V+}(x)-\bar{h}_{V+}(x) \not D \frac{1}{2 m+i D \cdot V} P_{V-} \not D h_{V+}(x),
$$


where $D^{\mu} \equiv \partial^{\mu}-i g_{s} A^{\mu}$ is the covariant derivative. This is the nonlocal form of the effective theory.

Obviously, this effective lagrangian depends on the velocity parameter $V$. The choice of this parameter is not unique. The RPI implies that physical predictions by the effective theory are independent of the choice of $V$. In Ref.[5], it was shown that the effective lagrangian (3) is invariant under an infinitesimal transformation $V \rightarrow V+\Delta V$

$$
\Delta h_{V+}(x)=\frac{\Delta V}{2}\left(h_{V+}(x)+h_{V-}(x)\right) .
$$

with the $h_{V-}(x)$ being the small component field and given by:

$$
h_{V-}(x)=\frac{1}{2 m+i D \cdot V} \mathrm{P}_{-} i \not D h_{V+}(x) .
$$

$\Delta V$ is constrained by $\Delta V \cdot V=0$ due to $V^{2}=1$.

Both the effective lagrangian (3) and the transformation given by (44) and (5) can be expanded as power series of $1 / m$. The RPI is then valid order by order in $1 / m$. It implies that the tree level transformation makes the tree level effective lagrangian valid at any order of $1 / \mathrm{m}$. The cancellation of the lagrangian shift $\Delta L$ at each order is quite nontrivial. Without expansion, the effective theory with the lagrangian (3) is equivalent to that of the full theory in the sense that they produce the same $S$-matrix elements.

\section{B. matching condition for renormalizing the transformation}

With expansion in terms of $1 / m$, the ultraviolet behavior of the theory is changed. To compensate this change, both the effective lagrangian and the transformation receive renormalization. The renormalization procedure of the effective lagrangian is well-known while it is not evident how to renormalize the transformation. The difficulty arises from the fact that each term contains the product of some covariant derivatives and the heavy quark field which is complicated and it is just the linear function of the heavy quark field but not bi-linear functions as those in the effective lagrangian.

Nevertheless, to gain the answer, it is instructive to recall the renormalization procedure of the effective lagrangian. For the heavy quark field defined in (1), there are simpler relations between Green's functions in the effective theory with the nonlocal form (3) and those in QCD full theory. For the expanded effective lagrangian, one has to add some local operators 
to the expanded effective lagrangian so that those relations can be satisfied. Consequently, the renormalized effective lagrangian can reproduce the results of full QCD. Therefore, those relations between the Green's functions are just the matching conditions to determine the effective lagrangian.

In order to renormalize the expanded transformation, the key point is to gain appropriate matching conditions to determine the transformation. As we will see, the renormalization of the transformation of the heavy quark field under the variation of the velocity parameter $V$ is attributed to the renormalization of the small component field in the proposed transformation (44). Therefore, if we can establish some relations between the Green's functions in QCD full theory and certain correlation functions which involve the $h_{V-}(x)$ field in the effective theory, they can be used as the matching conditions to renormalize the $h_{V-}(x)$ and hence the transformation. It is found that these relations can be obtained by imposing an infinitesimal transformation of $V$ on both sides of the relations between the Green's functions in QCD full theory and those in the effective theory.

Now let's first look at the relations between the Green's functions in the full QCD and those in the effective theory.

Denote the Green's function in the full theory by $G(x, y ; B)$ and that in the effective theory by $G_{V}(x, y ; B)$, respectively, where $B$ is an arbitrary background field. They are defined by

$$
G(x, y ; B) \equiv\langle 0|T \Psi(x) \bar{\Psi}(y)| 0\rangle^{B},
$$

and

$$
G_{V}(x, y ; B) \equiv\left\langle 0\left|T h_{V+}(x) \bar{h}_{V+}(y)\right| 0\right\rangle^{B}
$$

Any interaction vertex with gluon can then be obtained by functional differentiating over $B(x)$. When the quark field $h_{V+}(x)$ is related to the field in the full theory by Eq.(1), the Green's functions satisfy the following simple relation (A derivation of this relation using generating functional method is given in Appendix A):

$$
G_{V}(x, y ; B) \doteq \mathrm{P}_{+} G(x, y ; B) \mathrm{P}_{+},
$$

here $\doteq$ means that we omit the phase factor $\exp [i m V \cdot(x-y)]$ and the renormalization constant $Z\left(m, \alpha_{s}(m)\right)$ which arises from the renormalization of the heavy quark field in the 
full theory and that in the effective theory. Both sides are valid to all orders of $1 / m$ and $\alpha_{s}$. This relation ensures that the $S$-matrix elements in the effective theory are identical to those in the full theory. The local effective theory is gained by expanding the nonlocal effective lagrangian density (31). This expansion changes the ultraviolet behavior of the nonlocal effective theory. To reproduce the result of the nonlocal effective theory, one needs to add local operators to the expanded effective lagrangian. The relation (8) is required to be satisfied as matching conditions to determine the coefficients of those local operators. Thus the relations between the Green's functions in the full theory and those in the effective theory can be used as the matching conditions to determine the effective lagrangian.

Below starting from these relations we derive the matching conditions to determine the renormalized transformation of the heavy quark field.

Obviously, these relations are valid for arbitrary $V$. It allows us impose an infinitesimal transformation $V \rightarrow V+\Delta V$ on both sides. It follows that:

$$
\Delta G_{V}(x, y ; B) \doteq \frac{\Delta V}{2} G(x, y ; B) \mathrm{P}_{+}+\mathrm{P}_{+} G(x, y ; B) \frac{\Delta V}{2} .
$$

Again the symbol $\doteq$ means we omit the phase factor and a term arises from its infinitesimal shift which is trivial under the transformation.

Given the definitions of the Green's functions in Eqs.(6),(17), we have the following unique solution of (9):

$$
\left\langle 0\left|T\left(\Delta h_{V+}(x) \bar{h}_{V+}(y)\right)\right| 0\right\rangle^{B} \doteq \frac{\Delta \forall}{2}\langle 0|T \Psi(x) \bar{\Psi}(y)| 0\rangle^{B} \mathrm{P}_{+} .
$$

Eq. (10) implies that $\Delta h_{V+}(x)$ is proportional to $\Delta V$. Thus we can generally write it as the following form

$$
\Delta h_{V+}(x)=\frac{\Delta V}{2}\left(\mathrm{P}_{+} h_{V+}^{\prime}(x)+\mathrm{P}_{-} h_{V-}^{\prime}(x)\right)
$$

Substituting it into (10), Eq. (10) is then decomposed into two equations by projection operator:

$$
\begin{aligned}
\left\langle 0\left|T\left(h_{V+}^{\prime}(x) \bar{h}_{V+}(y)\right)\right| 0\right\rangle^{B} & \doteq \mathrm{P}_{+}\langle 0|T \Psi(x) \bar{\Psi}(y)| 0\rangle^{B} \mathrm{P}_{+} \\
\left\langle 0\left|T\left(h_{V-}^{\prime}(x) \bar{h}_{V+}(y)\right)\right| 0\right\rangle^{B} & \doteq \mathrm{P}_{-}\langle 0|T \Psi(x) \bar{\Psi}(y)| 0\rangle^{B} \mathrm{P}_{+}
\end{aligned}
$$

Eq. (12) is nothing but Eq. (8) if $h_{V+}^{\prime}(x)$ is identical to $h_{V+}(x)$. Eq. (13) is a new one which can be regarded as the definition of the $h_{V-}^{\prime}(x)$. Some comments can be given about this 
equation. The right hand side is still the Green's functions in the full theory while the left hand side is the Green's functions in the effective theory with insertion of local operators at point $x$. Again both sides are valid at any desired order of $1 / m$ and $\alpha_{s}$ with all possible interaction with gluons.

For the effective lagrangian at tree level, this equation is satisfied if $\Delta h_{V+}(x)$ is given by transformation (4) with $h_{V-}(x)$ given by the expanded expression (15). At loop level, it is also valid at any specific loop momentum which is smaller than the quark mass. However, in calculating the whole loop momentum integration, some differences arise and the (13) can be regarded as the matching condition to determine the renormalized $h_{V-}(x)$ field.

Eq. (11) together with (12) and (13) imply that the renormalized transformation keeps the same form as the tree-level transformation (4). But only the small component field needs to be renormalized.

Now let's illustrate how (13) determine the renormalized $h_{V-}(x)$ field as the matching conditions. For simplicity, we limit our arguments in the hard cutoff regularization. Similar arguments are applicable to dimensional regularization.

Suppose one takes different hard cut-off regularization energy scales $\Lambda_{e}$ and $\Lambda_{f}$ in effective theory and in full theory, respectively. The $\Lambda_{f}$ should be much larger than the heavy quark mass $m$ for including both quark and antiquark contributions. The $\Lambda_{e}$ should be much smaller than $m$ for the validation of the $1 / m$ expansion. So they satisfy a hierarchy relation $\Lambda_{e} \ll m \ll \Lambda_{f}$. Thus in calculating loop diagrams the integration bounds are different on both sides ( the left hand side is integrated out from 0 to $\Lambda_{e}$ while the right hand side is integrated from 0 to $\left.\Lambda_{f}\right)$. It leads to that the tree level expression of $h_{V-}(x)$ given by the expansion of (5) makes relation (13) no longer valid. To compensate the differences, one has to add the contributions of the loop momentum integrals from $\Lambda_{e}$ to $\Lambda_{f}$ to the left hand side. Those contributions can be expressed as the insertions of the local operators on the heavy quark line. They may be at point $x$ or not. Those local operators at point $x$ can be absorbed into the redefinition of the $h_{V-}^{\prime}(x)$ while those local operators not shrunk at the point $x$ correspond to the insertions of the higher dimensional operators in the effective lagrangian. Therefore, Eq. (11) is just the matching conditions for determining the renormalized small component field $h_{V-}(x)$. Eq. (13) allows one determine the renormalized $h_{V-}(x)$ to any order of $1 / m$ and $\alpha_{s}$. 


\section{RENORMALIZED TRANSFORMATION TO ORDER $1 / \mathrm{m}^{2}$}

As a specific example, in this section, we determine the renormalized effective lagrangian and the $h_{V-}(x)$ field up to the next leading order corrections of $1 / m$ using the matching conditions (8) and (13), respectively. Here we use the dimensional regularization and Feynman gauge in all specific calculations.

Up to next leading order corrections of $1 / m$, the most general form of the renormalized effective lagrangian can be expressed as:

$$
\begin{aligned}
L_{V}^{1}(x)= & Z \bar{h}_{V+}(x) i D \cdot V h_{V+}(x)+\frac{Z}{2 m} \bar{h}_{V+}(x) \mathbf{D}^{2} h_{V+}(x) \\
& +\frac{Z Z_{e}}{2 m} \bar{h}_{V+}(x)(i D \cdot V)^{2} h_{V+}(x)+\frac{Z Z_{m}}{4 m} h_{V+}(x) \sigma^{\mu \nu} G_{\mu \nu}(x) h_{V+}(x),
\end{aligned}
$$

$\sigma^{\mu \nu} \equiv \frac{i}{2}\left[\gamma^{\mu}, \gamma^{\nu}\right]$, and the most general form of the renormalized $h_{V-}(x)$ field can be written as:

$$
h_{V-}(x)=\mathrm{P}_{-}\left(\frac{d_{0}(\mu)}{2 m} i \not D+\frac{d_{1}(\mu)}{4 m^{2}} D \cdot V \not D+\frac{d_{2}(\mu)}{4 m^{2}} \not D D \cdot V\right) h_{V+}(x) \text {, }
$$

where $Z, Z_{e}, Z_{m}, d_{0}(\mu), d_{1}(\mu), d_{2}(\mu)$ are the short distance coefficients to be determined.

In the following matching procedures, all the short-distance coefficients are assumed to be calculated to all orders in $\alpha_{s}$, which makes the matching procedure are applicable for higher order calculations. But in this paper we only present one-loop result in the final expression.

Since the off-shell momenta of the heavy quark and the momenta of the gluons can be treated as much smaller than the quark mass, in the matching procedure, the integrand can be expanded as power series of these momenta over the heavy quark mass. It leads to that the remainder part of the loop momentum integrals no longer depends on the heavy quark mass. We are free to choose the infrared regulator since the infrared divergences cancel on both sides. If we use a limitation order in which the external off-shell momenta of heavy quark and the momenta of the gluons go to zero first, followed by $\epsilon=2-D / 2$ going to zero, as used by Eichten and Hill in Ref. 1], then all terms such as $k^{\epsilon}$ arising from the loop momentum integrals vanish. In the effective theory, this implies that all contributions from the loop diagrams vanishes since all loop momentum integrals are proportional to it while in the full theory it implies that there is no any logarithmic nonlocal terms of these external momenta. This simplifies the matching calculations significantly.

It is easy to see that to determine these coefficients, we need to match both the two-point and three-point functions. 


\section{A. matching two-point function}

Let's first see what we can learn from matching the two-point function. For external momentum $p$ of the heavy quark near mass-shell, the general form of the QCD heavy quark self-energy, the inverse of the two-point function, with above infrared regulator can be written as:

$$
\Sigma(p)=A(\not p-m)+2 B m \Delta,
$$

where $\Delta$ is defined as

$$
\Delta \equiv \frac{p^{2}-m^{2}}{4 m^{2}} .
$$

The heavy quark expansion implies that $\Delta \ll 1$. Thus $A, B$ can be expanded as power series of $\Delta$. Up to next leading order, they can be written as:

$$
\begin{aligned}
& A=1+c_{0}(\mu)+c_{2}(\mu) \Delta, \\
& B=c_{1}(\mu)+c_{4}(\mu) \Delta .
\end{aligned}
$$

At one-loop level in QCD, there is only one 1PI diagram contributing to the self-energy, as shown in Fig. 1. Carrying out a specific calculation with the above infrared regulator, we obtain that:

$$
\begin{array}{ll}
c_{0}(\mu)=\frac{C_{F}}{4 \pi} \alpha_{s}(\mu)\left(\ln \frac{\mu^{2}}{m^{2}}+2\right), & c_{1}(\mu)=\frac{C_{F}}{2 \pi} \alpha_{s}(\mu)\left(\ln \frac{\mu^{2}}{m^{2}}+1\right), \\
c_{2}(\mu)=-\frac{C_{F}}{\pi} \alpha_{s}(\mu)\left(\ln \frac{\mu^{2}}{m^{2}}+2\right), & c_{4}(\mu)=-\frac{C_{F}}{\pi} \alpha_{s}(\mu)\left(\ln \frac{\mu^{2}}{m^{2}}-1\right),
\end{array}
$$

with $C_{F}=4 / 3$.

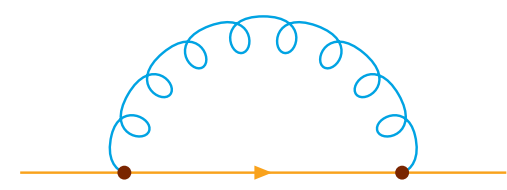

Fig. 1 Self-energy diagram on QCD side.

The two-point Green's function reads:

$$
\begin{aligned}
G(p) & =\frac{i}{\Sigma(p)} \\
& =i \frac{A(\not p+m)-2 B m \Delta}{4 m^{2} \Delta\left(A^{2}+A B-B^{2} \Delta\right)} .
\end{aligned}
$$


Now we first determine the effective lagrangian up to order $1 / m$ corrections using the matching condition (8). With (20), the QCD side of (8) reads:

$$
\begin{aligned}
\mathrm{P}_{+} G(p) \mathrm{P}_{+} & =i \frac{A(2 m+k \cdot V)-2 B m \Delta}{4 m^{2} \Delta\left(A^{2}+A B-B^{2} \Delta\right)} \mathrm{P}_{+} \\
& =\frac{i}{c k \cdot V}\left(1+\frac{\mathbf{k}^{2}}{2 m k \cdot V}-\frac{c_{1}+c_{2}+c_{4}}{c} \delta\right) \mathrm{P}_{+},
\end{aligned}
$$

where $\delta \equiv k \cdot V / 2 m$ and $c=1+c_{0}+c_{1}$.

As we argued above, on the side of the effective theory, contributions from the loop diagrams vanish. Thus all contributions to the right side of the matching condition (8) arise from the tree diagrams. With all possible insertion of higher order terms, the right side of the matching condition (8) reads:

$$
\frac{i}{Z k \cdot V}\left(1+\frac{\mathbf{k}^{2}}{2 m k \cdot V}-Z_{e} \delta\right) \mathrm{P}_{+} .
$$

Comparing (22) to (21), we see that $Z=c=1+c_{0}+c_{1}$, and $Z_{e}=\left(c_{1}+c_{2}+c_{4}\right) / c$. With the one-loop values given in (19), we have:

$$
\begin{aligned}
Z & =1+\frac{C_{F}}{4 \pi} \alpha_{s}(\mu)\left(3 \ln \frac{\mu^{2}}{m^{2}}+4\right), \\
Z_{e} & =-\frac{C_{F}}{2 \pi} \alpha_{s}(\mu)\left(3 \ln \frac{\mu^{2}}{m^{2}}+1\right) .
\end{aligned}
$$

These results are in agreement with those presented in literatures. The coefficient $Z_{m}$ can only be determined by matching the 3-point function in the next subsection.

We then use the matching condition (13) to determine the renormalized $h_{V-}(x)$ field up to order $1 / m$ corrections. With the two-point function given in (20), the QCD side of (13) reads:

$$
\begin{aligned}
\mathrm{P}_{-} G(p) \mathrm{P}_{+} & =i \frac{A \mathrm{P}_{-} \not k \mathrm{P}_{+}}{4 m^{2} \Delta\left(A^{2}+A B-B^{2} \Delta\right)} \\
& =i \frac{\mathrm{P}_{-} \not k \mathrm{P}_{+}}{2 m c k \cdot V}\left[1+\frac{\mathbf{k}^{2}}{2 m k \cdot V}-\left(1+\frac{c_{2}+c_{4}}{c}-\frac{c_{1}^{2}}{c\left(1+c_{0}\right)}\right) \delta\right] .
\end{aligned}
$$

On the effective theory side, again contributions from loop diagrams vanish while only tree diagrams survive. Up to next leading order correction terms five diagrams give nonzero contributions, as shown in Fig.2. The Feynman rules for the operator insertions in these diagrams can easily be obtained from (14) and (15). Their contributions to the right side of (13) reads:

$$
i \frac{\mathrm{P}_{-} \not k \mathrm{P}_{+}}{2 m}\left[\frac{1}{Z k \cdot V}+\frac{\mathbf{k}^{2}}{2 m Z(k \cdot V)^{2}}-\left(\frac{Z_{e}}{2 m Z}+\frac{d_{1}+d_{2}}{2 m Z}\right) \delta\right] .
$$



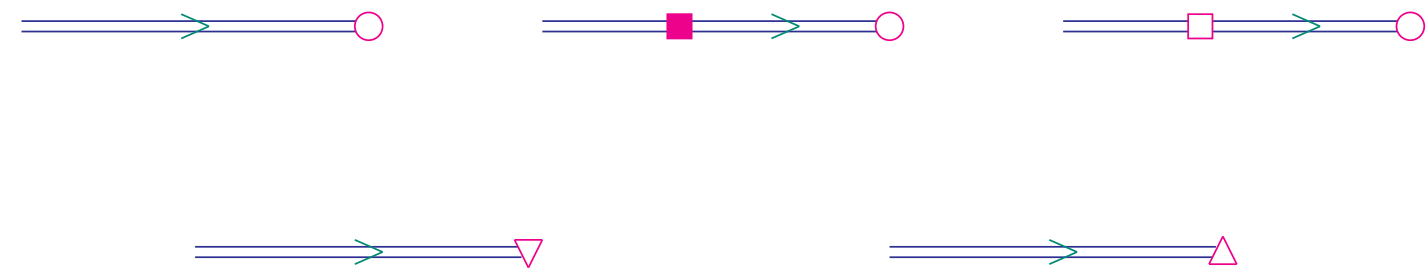

Fig. 2 Diagrams contributing to the matching conditions on the effective theory side. The circle, up and down triangles represent the operators with coefficient $d_{0}$, $d_{1}$, and $d_{2}$, respectively, while the solid and the blank boxes represent the insertion of the kinetic and $(D \cdot V)^{2} / 2 m$ operators in the effective lagrangian.

Comparing (24) to (25), we obtain that:

$$
\begin{aligned}
d_{0} & =1, \\
d_{1}+d_{2} & =1-\frac{c_{1}}{1+c_{0}} .
\end{aligned}
$$

We see that only the combination of the $d_{1}$ and $d_{2}$ can be determined by the matching through the 2-point function. To determine them separately, we need to match the 3-point function.

\section{B. matching three-point function}

In this subsection, we determine the short-distance coefficients $Z_{m}$ in (14) and $d_{1}, d_{2}$ in (15) by matching the three-point function. Here the Feynamn diagrams with 3-gluon vertex

are involved. We use the background field method [14], which can be used to simplify the calculations significantly. In this method, QCD Ward identity for the self-energy and the 1PI quark-gluon vertex takes a QED-like form:

$$
k_{\mu} \Gamma^{\mu}\left(p_{1}, p_{2}\right)=\Sigma\left(p_{2}\right)-\Sigma\left(p_{1}\right),
$$

where $\Gamma^{\mu}\left(p_{1}, p_{2}\right)$ is the $1 \mathrm{PI} 3$-vertex with external quark momenta $p_{1}$, and $p_{2}$, and gluon momentum $k=p_{2}-p_{1}$.

Up to next leading order correction terms, the general form of the QCD 1PI vertex satisfying the Ward identity (27) with quark near threshold can be written as:

$$
\Gamma^{\mu}\left(p_{1}, p_{2}\right)=\bar{A} \gamma^{\mu}+\frac{\bar{B}}{m} \bar{p}^{\mu}+\frac{c_{2}}{2 m^{2}} \bar{p}^{\mu}(\not \vec{p}-m)+\frac{c_{3}}{4 m}\left[\not k, \gamma^{\mu}\right],
$$


where

$$
\begin{aligned}
\bar{A} & =1+c_{0}+c_{2} \bar{\Delta} \\
\bar{B} & =c_{1}+2 c_{4} \bar{\Delta} \\
\bar{p} & \equiv \frac{1}{2}\left(p_{1}+p_{2}\right) \\
\bar{\Delta} & \equiv \frac{p_{1}^{2}+p_{2}^{2}-2 m^{2}}{8 m^{2}}
\end{aligned}
$$

The one-loop coefficients $c_{0}, c_{1}, c_{2}$, and $c_{4}$ have been given in (19). Thus we only need to evaluate the $c_{3}$. For simplicity, we take the polarization vector of the gluon $e$ satisfying:

$$
e \cdot p_{1}=e \cdot p_{2}=e \cdot k=0
$$

Then we have:

$$
\Gamma^{e}\left(p_{1}, p_{2}\right) \equiv \Gamma^{\mu}\left(p_{1}, p_{2}\right) e_{\mu}=\bar{A} \notin+\frac{c_{3}}{4 m}[\not k, \notin]
$$

In QCD, at one-loop level, there are two Feynman diagrams contributing to $\Gamma^{e}\left(p_{1}, p_{2}\right)$ as shown in Fig. 3. A straightforward calculation gives that:

$$
c_{3}(\mu)=\left(2 C_{F}+C_{A}\right) \frac{\alpha_{s}(\mu)}{4 \pi}\left(\ln \frac{\mu^{2}}{m^{2}}+2\right) .
$$
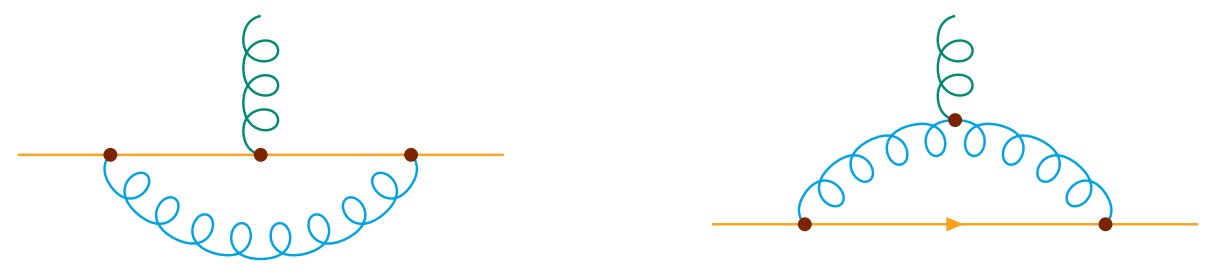

Fig. 3 Vertex diagrams on QCD side

In the matching procedure, taking the polarization vector $e$ can also significantly simplifies the calculations. In QCD, the general form of the 3-point Green's function with this vertex contributing to the matching conditions (18) and (13) is expressed by:

$$
G^{e}\left(p_{1}, p_{2}\right)=G\left(p_{1}\right) \Gamma^{e}\left(p_{1}, p_{2}\right) G\left(p_{2}\right)
$$


Now we first determine $Z_{m}$ by using (8). The QCD side of (8) reads:

$$
\begin{aligned}
& \mathrm{P}_{+} G\left(p_{1}\right) \Gamma^{e}\left(p_{1}, p_{2}\right) G\left(p_{2}\right) \mathrm{P}_{+} \\
&=- \mathrm{P}_{+} \frac{A_{1}\left(\not \ell_{1}+2 m\right)-2 B_{1} m \Delta}{4 m^{2} \Delta_{1}\left(A_{1}^{2}+A_{1} B_{1}-B_{1}^{2} \Delta_{1}\right)}\left(\bar{A} \notin+\frac{c_{3}}{4 m}[\not k, \phi]\right) \\
& \quad \times \frac{A_{2}\left(\not k_{2}+2 m\right)-2 B_{2} m \Delta_{2}}{4 m^{2} \Delta_{2}\left(A_{2}^{2}+A_{2} B_{2}-B_{2}^{2} \Delta_{2}\right)} \mathrm{P}_{+},
\end{aligned}
$$

where the subscript 1 and 2 denote the momentum being $p_{1}$ and $p_{2}$, respectively. Expanding it to leading order of $k$, we have

$$
-\frac{1}{4 m c^{2} k_{1} \cdot V k_{2} \cdot V}\left(1+c_{0}+c_{3}\right) \mathrm{P}_{+}[\not k, \notin] \mathrm{P}_{+} .
$$

On the effective theory side, only the insertion of the color- magnetic dipole term gives non-zero contribution for the gluon polarization vector satisfying (301). It reads:

$$
-\frac{Z_{m}}{4 m Z k_{1} \cdot V k_{2} \cdot V} \mathrm{P}_{+}[\not k, \notin] \mathrm{P}_{+} .
$$

Comparing (35) to (36), we determine that

$$
Z_{m}=\frac{1+c_{0}+c_{3}}{c}=1+\frac{c_{3}-c_{1}}{c} .
$$

With the coefficients given in (19) and (32), $Z_{m}$ at one-loop level reads:

$$
Z_{m}=1+\frac{\alpha_{s}(\mu)}{4 \pi}\left(C_{A} \ln \frac{\mu^{2}}{m^{2}}+2 C_{A}+2 C_{F}\right) .
$$

We then determine $d_{1}$ and $d_{2}$ in the renormalized $h_{V}$ - field (15) by using the matching condition (13). The QCD side of (13) reads:

$$
\begin{aligned}
& \mathrm{P}_{-} G\left(p_{1}\right) \Gamma^{e}\left(p_{1}, p_{2}\right) G\left(p_{2}\right) \mathrm{P}_{+} \\
= & -\mathrm{P}_{-} \frac{A_{1}\left(\not \ell_{1}+2 m\right)-2 B_{1} m \Delta}{4 m^{2} \Delta_{1}\left(A_{1}^{2}+A_{1} B_{1}-B_{1}^{2} \Delta_{1}\right)}\left(\bar{A} \notin+\frac{c_{3}}{4 m}[\not k, \not]\right) \\
& \times \frac{A_{2}\left(\not k_{2}+2 m\right)-2 B_{2} m \Delta_{2}}{4 m^{2} \Delta_{2}\left(A_{2}^{2}+A_{2} B_{2}-B_{2}^{2} \Delta_{2}\right)} \mathrm{P}_{+},
\end{aligned}
$$

The expression can be expanded as power series of $k$ 's. Keeping only the leading corrections, (39) reads:

$$
\begin{aligned}
& \mathrm{P}_{-} \notin \mathrm{P}_{+} \frac{1}{2 m c k_{2} \cdot V}\left[1+\frac{\mathbf{k}_{2}^{2}}{2 m k_{2} \cdot V}\right. \\
& \left.-\left(1-\frac{2 c_{1}-c_{2}-2 c_{3}}{2\left(1+c_{0}\right)}\right) \delta_{1}-\frac{c_{1}+c_{2}+c_{4}}{c} \delta_{2}+\frac{c_{2}+2 c_{3}}{2\left(1+c_{0}\right)} \delta_{2}\right] \\
& -\frac{1+c_{0}+c_{3}}{4 m c^{2}} \frac{1}{k_{1} \cdot V k_{2} \cdot V} \mathrm{P}_{-} \not k \mathrm{P}_{+}[\not k, \not] \mathrm{P}_{+} .
\end{aligned}
$$


On the effective theory side, contributions may arise the insertions of the operators both in $h_{V-}(x)$ given in (15) and in the effective lagrangian given in (14). With the polarization vector $e$, there are 6 Feynman diagrams contributing to it as shown in Fig.4. With appropriate Feynman rules, they read:

$$
\begin{aligned}
& \mathrm{P}_{-} \notin \mathrm{P}_{+} \frac{1}{2 m Z k_{2} \cdot V}\left[1+\frac{\mathbf{k}_{2}^{2}}{2 m k_{2} \cdot V}-d_{1} \delta_{1}-\frac{Z_{e}}{Z} \delta_{2}-d_{2} \delta_{2}\right] \\
& -\frac{Z_{m}}{4 m Z} \frac{1}{k_{1} \cdot V k_{2} \cdot V} \mathrm{P}_{-} \not k \mathrm{P}_{+}[\not k, \notin] \mathrm{P}_{+} .
\end{aligned}
$$
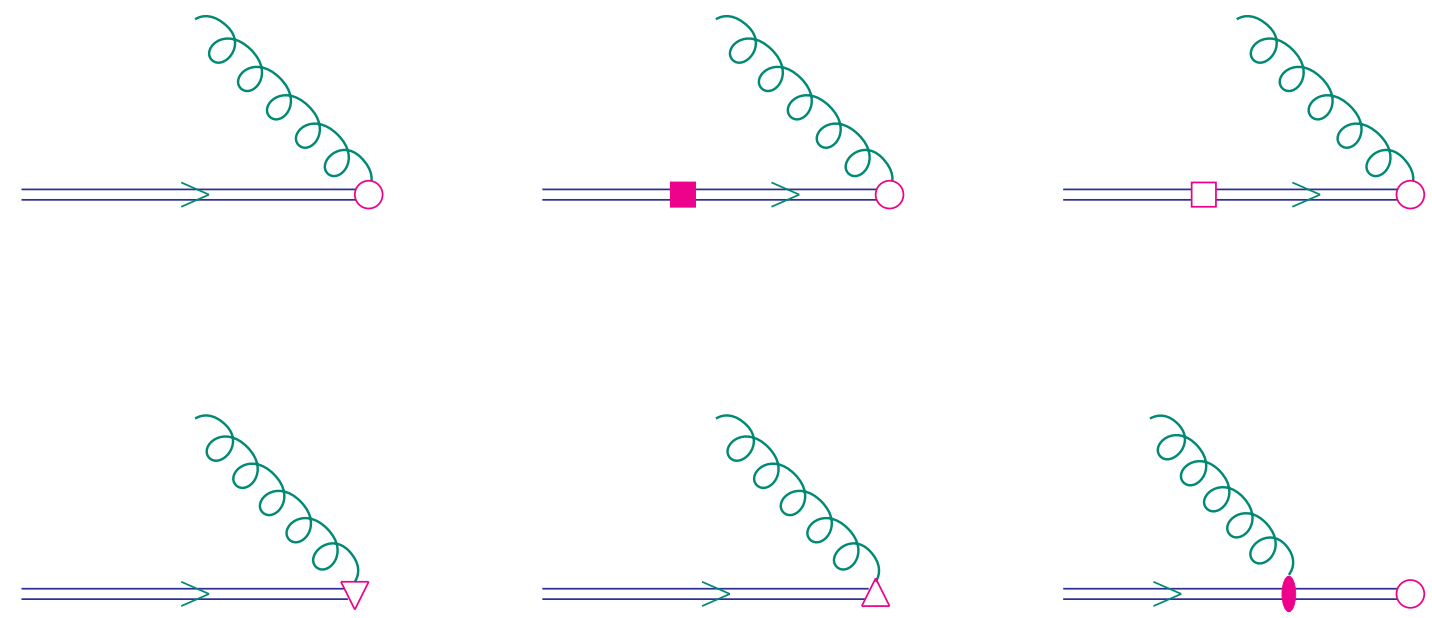

Fig. 4 Diagrams contributing to the matching conditions on the effective theory side. The notations are the same as figure 2. The solid Oval represents the insertion of the color-magnetic dipole operator.

Comparing (39) to (41), we determine that

$$
\begin{aligned}
& d_{1}=1-\frac{2 c_{1}-c_{2}-2 c_{3}}{2\left(1+c_{0}\right)}, \\
& d_{2}=-\frac{c_{2}+2 c_{3}}{2\left(1+c_{0}\right)} .
\end{aligned}
$$

These values are consistent with (26) obtained by matching the 2-point function.

Substituting the short-distance coefficients in (19) and (32) into (42), we obtain the one-loop renormalized coefficients for $d_{1}$ and $d_{2}$ :

$$
\begin{aligned}
& d_{1}(\mu)=1+\frac{\alpha_{s}(\mu)}{4 \pi}\left[\left(C_{A}-2 C_{F}\right) \ln \frac{\mu^{2}}{m^{2}}-2 C_{F}+2 C_{A}\right] \\
& d_{2}(\mu)=-\frac{\alpha_{s}(\mu)}{4 \pi} C_{A}\left(\ln \frac{\mu^{2}}{m^{2}}+2\right)
\end{aligned}
$$


with $C_{A}=3$. These are the central results of this section. These results are in disagreement with that obtained by Balzereit in [9]. Since we use the same definition of the heavy quark field, both result should be equal. The results presented in this paper are derived rigorously using the matching conditions while Balzereit obtained indirectly from the requirement of the invariance of the effective lagrangian which is much more complicated.

\section{RPI OF THE RENORMALIZED EFFECTIVE LAGRANGIAN}

In this section, I compare the renormalized transformation determined by the matching condition (13) with those given in 7]. I show that their results can easily be understood by constructing the effective lagrangian in an alternative way, in which an effective theory in four-component field is constructed first, followed by its reduction to the effective theory in two-component field. I then prove that the renormalized transformation determined by the matching conditions (13) can be written as the same form with the transformation (41) and (5) with the covariant derivative substituted by the operator which may be called as the generalized covariant derivative. It means that the result presented in this paper is consistent with that given in [7]. Finally, I will show that the renormalized effective lagrangian is reparameterization invariant under the renormalized transformation.

\section{A. effective lagrangian in four-fermion field}

In the conventional method, a renormalized effective lagrangian is constructed by following steps. First a proper field to describe the low energy particles is chosen. In HQET and NRQCD, this effective field for describing the the heavy quark is just the two-component field. Then the effective lagrangian in this field is expanded as sum of local operators in terms of appropriate counting rules. Then the renormalized short distance coefficients of these local operators are determined by matching the full theory and the effective theory. We refer this method as matching after expansion.

Here we introduce an alternative way to determine the renormalized effective lagrangian. In this method, renormalized local operators expressed in the field of the full theory are added to the lagrangian of the full theory by matching conditions. Then it is expanded in terms of the two-component field. We refer this method as matching before expansion. 
Let's illustrate how this works in a hard cut-off regularization.

As in the last section, we take different hard cut-off regularization energy scales $\Lambda_{e}$ and $\Lambda_{f}$ in the effective theory and in the full theory, respectively. They satisfy $\Lambda_{e} \ll m \ll \Lambda_{f}$. In calculating the one-loop 1PI diagrams in full QCD theory, we need to calculate the loop momentum integrals from zero to $\Lambda_{f}$. They can be separated into integrals from 0 to $\Lambda_{e}$ and integrals from $\Lambda_{e}$ to $\Lambda_{f}$. The first part is just the same with that in the effective theory while the second part gives extra contributions. As argued above, the contributions from this region can be written as local terms of external momenta and can be expressed as contributions from local operators. Therefore, once those local operators are added to the lagrangian, the effective theory with hard cutoff $\Lambda_{e}$ can produce the same result of the full theory with cutoff $\Lambda_{f}$. This argument can easily be generalized to the case of multi-loops.

At this stage, those local operators are written in terms of Dirac four-component field. A general form of the renormalized effective lagrangian density with hard cutoff $\Lambda_{e}$ for heavy quark field can formally be expressed as:

$$
\mathcal{L}_{\mathrm{eff}}=\bar{\Psi}(x)(i \not D-m) \Psi(x)+\bar{\Psi}(x) O_{1}(x) \Psi(x)
$$

where $D^{\mu}=\partial^{\mu}-i g A_{\mu}^{a} T^{a}$ is the covariant derivative. It may be denoted as

$$
\mathcal{L}_{\text {eff }}=\bar{\Psi}(x) O(x) \Psi(x),
$$

for short by defining $O(x) \equiv i \not D-m+O_{1}(x)$.

The first term in (45) is just the tree-level lagrangian while the second term arises from the renormalization with a cut-off $\Lambda_{e} \ll m$. The operators in this term are generally the function of the covariant derivative and the heavy quark mass. It may contain terms such as $D^{2}+m^{2}$, and $g_{s} G^{\mu \nu}=i\left[D^{\mu}, D^{\nu}\right]$, which are suppressed by the off-shell momentum of the heavy quark or the momenta of the external gluons. They can be organized via appropriate power counting rules. In perturbative calculations, the loop momentum integral is from zero to $\Lambda_{e}$. In this region, both the external and the loop momenta are smaller than $m$, hence the $1 / m$ expansion is allowed and the quark mass dependence is extracted explicitly. Thus the energy scale $m$ is no longer involved in the effective theory. I emphasis here that the effective lagrangian density in this form is independent of the velocity parameter $V$. Thus it automatically satisfies the RPI.

Higher dimensional operators appear in $O_{1}(x)$. It implies that power divergences arise in the loop momentum integrals. In the full theory the power divergences cancel when both the 
contributions from quark and antiquark are included. However, in the effective theory when we impose a hard cutoff $\Lambda_{e} \ll m$ on the loop momentum integrals, the contributions from antiquark are excluded so that the power divergences do not cancel. Nevertheless, those power divergences are artificial since the power divergences from the diagram calculations just cancel those from the short distance coefficients.

At one loop and leading order of $1 / m$, the most general form of the four-component effective lagrangian is

$$
\begin{aligned}
\mathcal{L}_{\mathrm{eff}}= & \left(1+c_{0}\right) \bar{\Psi}(x)(i \not D-m) \Psi(x)-\frac{c_{1}}{2 m} \bar{\Psi}(x)\left(D^{2}+m^{2}\right) \Psi(x) \\
& -\frac{i c_{2}}{8 m^{2}} \bar{\Psi}(x)\left[(i \not D-m)\left(D^{2}+m^{2}\right)+\left(D^{2}+m^{2}\right)(i \not D-m)\right] \Psi(x) \\
& +\frac{c_{3}}{4 m} \bar{\Psi}(x) g_{s} \sigma^{\mu \nu} G_{\mu \nu} \Psi(x)+\frac{c_{4}}{8 m^{3}} \bar{\Psi}(x)\left(D^{2}+m^{2}\right)^{2} \Psi(x) .
\end{aligned}
$$

Calculating the the 1PI diagrams shown in Fig. 1 and Fig 3 using this effective lagrangian and full QCD, we see that these coefficients are just those $c_{1}-c_{4}$ given in (19)), and (32).

\section{B. effective lagrangian in two-component field}

Now let's reduce (47) to the effective lagrangian in two-component field. The equation of motion now reads:

$$
\mathrm{P}_{-} \bar{O}(x)\left(h_{V+}(x)+h_{V-}(x)\right)=0,
$$

where $\bar{O}(x)$ is the $O(x)$ whose covariant derivative $i D$ is replaced by $i D+m V$ due to the phase factor in the field redefinition. It can be regarded as the renormalized equation of motion.

From Eq.(48), we can express $h_{V-}(x)$ as a function of $h_{V+}(x)$ formally as:

$$
h_{V-}(x)=\frac{1}{2 m+i D(x) \cdot V-\mathrm{P}_{-} \bar{O}_{1}(x) \mathrm{P}_{-}} \mathrm{P}_{-}\left(i \not D+\bar{O}_{1}(x)\right) h_{V+}(x),
$$

where $\bar{O}_{1}(x)$ is the $O_{1}(x)$ whose covariant derivative $i D$ is substituted by $i D+m V$. This modifies the tree level expression (15). Once the form of $O_{1}(x)$ is given, the right hand side of Eq.(49) can be expanded as power series of $1 / m$. With $O_{1}(x)$ given in (47), up to order $\alpha_{s}$ and $1 / m^{2}, h_{V-}(x)$ reads:

$$
\begin{aligned}
h_{V-}(x)= & \mathrm{P}_{-}\left[\frac{1}{2 m} i \not D+\frac{1}{4 m^{2}}\left(1-\frac{2 c_{1}-c_{2}-2 c_{3}}{2\left(1+c_{0}\right)}\right) D \cdot V \not D\right. \\
& \left.-\frac{c_{2}+2 c_{3}}{8\left(1+c_{0}\right) m^{2}} \not D D \cdot V\right] h_{V+}(x) .
\end{aligned}
$$


Comparing this with (15), (42), we see that they are in agreement.

Finally, with equation of motion (48), the effective lagrangian (46) is reduced to:

$$
\begin{aligned}
\mathcal{L}_{\mathrm{eff}} & =\bar{h}_{V+}(x) \bar{O}(x)\left(h_{V+}(x)+h_{V-}(x)\right) \\
& =\left(\bar{h}_{V+}(x)+\bar{h}_{V-}(x)\right) \bar{O}(x) h_{V+}(x) .
\end{aligned}
$$

This is just the two-component effective lagrangian. It can be expanded as power series of $1 / m$. In this way, the four component effective lagrangian is reduced to the two component effective lagrangian. Up to order $\alpha_{s}$ and $1 / m$ correction, it reduced to the effective lagrangian (14). Therefore, the effective lagrangian obtained by these two different approaches are just the same.

\section{C. comparison with the previous work}

In Sec. II, the matching condition for determining the renormalized $h_{V-}^{\prime}(x)$ field is given by Eq. (13). In the last subsection, the $h_{V-}(x)$ field has been obtained by equation of motion. Its expression is given by Eq. (49). In this subsection, I will show that the small component fields obtained by these two different methods are identical. They uniquely determine the renormalized transformation of the heavy quark field against the infinitesimal variation of the velocity parameter $V$. Adding both sides of (12) and (13) together, we have

$$
\left\langle 0\left|T\left(h_{V+}(x)+h_{V-}^{\prime}(x)\right) \bar{h}_{V+}(y)\right| 0\right\rangle^{B} \doteq\langle 0|T \Psi(x) \bar{\Psi}(y)| 0\rangle^{B} \mathrm{P}_{+} .
$$

where $\langle 0|T \Psi(x) \bar{\Psi}(y)| 0\rangle^{B}$ is a full propagator under arbitrary external field $B^{\mu}(x)$. It is satisfied order by order in $\alpha_{s}$. Suppose we calculate the left hand side at tree level with the renormalized effective lagrangian. To validate this equation, the right hand side then should also be calculated to the tree level with containing contributions of the loop momentum integrals from $\Lambda_{e}$ to $\Lambda_{f}$. This can be calculated by the renormalized four-component effective lagrangian (45) to tree level. Thus it satisfies the following equation

$$
O(x) G(x, y ; B)=i \delta^{4}(x-y)
$$

Acting an operator $\mathrm{P}_{-} \bar{O}(x)$ on the left hand side and $\mathrm{P}_{-} O(x)$ on the right hand side of (52), the right hand side vanishes immediately since $\mathrm{P}_{-} \cdot \mathrm{P}_{+}=0$. Since we only calculate them at tree level, the operator $\bar{O}(x)$ can be moved within the bracket:

$$
\mathrm{P}_{-}\left\langle 0\left|T\left[O(x)\left(h_{V+}(x)+h_{V-}^{\prime}(x)\right) \bar{h}_{V+}(y)\right]\right| 0\right\rangle^{B}=0 .
$$


Since the argument $y$ in $\bar{h}_{V+}(y)$ is arbitrary and this correlation function contains interaction with arbitrary background gluon field, the unique solution of this equation is

$$
\mathrm{P}_{-} \bar{O}(x)\left(h_{V+}(x)+h_{V-}^{\prime}(x)\right)=0 .
$$

This is just identical to (48) if $h_{V-}^{\prime}(x)$ is the same with $h_{V-}(x)$. This implies that the

renormalized $h_{V-}^{\prime}(x)$ determined by the matching condition (13) is identical to that from the equation of motion (48).

\section{RPI of the renormalized effective lagrangian}

In this subsection, let's prove that the renormalized effective lagrangian (151) is invariant under the transformation (4) or (11) with renormalized small component field.

It follows that from an infinitesimal transformation of the effective lagrangian (51)

$$
\begin{aligned}
\Delta \mathcal{L}_{\mathrm{eff}} & \doteq \Delta \bar{h}_{V+}(x) \bar{O}(x) h_{V}(x)+\bar{h}_{V+}(x) \bar{O}(x) \Delta h_{V}(x) \\
& =\bar{h}_{V}(x) \frac{\Delta V}{2} \bar{O}(x) h_{V}(x)+\bar{h}_{V+}(x) \bar{O}(x) \Delta h_{V}(x)
\end{aligned}
$$

We have use a shorthand notation $h_{V}(x)=h_{V+}(x)+h_{V-}(x)$. It is emphasized here that the operator $O(x)$ which is from the four component effective field theory is invariant against the variation of the velocity $V$. Any change arising from the phase factor in the definition of the effective field has been omitted simply because it is trivial under the transformation.

Imposing an infinitesimal transformation on the equation of motion (48), we obtain that

$$
-\frac{\Delta \forall}{2} \bar{O}(x) h_{V}(x)+\mathrm{P}_{-} \bar{O}(x) \Delta h_{V}(x) \doteq 0
$$

With it, (56) can be rewritten as

$$
\Delta \mathcal{L}_{\mathrm{eff}} \doteq \bar{h}_{V}(x) \bar{O}(x) \Delta h_{V}(x)
$$

Notice that $\mathrm{P}_{+} h_{V-}(x)=0$. Imposing an infinitesimal on it, we immediately have

$$
\mathrm{P}_{+} \Delta h_{V-}(x)=-\frac{\Delta V}{2} h_{V-}(x)
$$

Adding it together with $\mathrm{P}_{+} \Delta h_{V+}(x)=\Delta V / 2 h_{V-}(x)$, we have

$$
\mathrm{P}_{+} \Delta h_{V}(x)=0
$$


With it, (56) is reduced to

$$
\Delta \mathcal{L}_{\text {eff }} \doteq \bar{h}_{V}(x) \bar{O}(x) \mathrm{P}_{-} \Delta h_{V}(x)
$$

It follows that $\Delta \mathcal{L}_{\text {eff }}=0$ from the equation of motion $\bar{h}_{V}(x) O(x) \mathrm{P}_{-}=0$. Thus we have shown that the renormalized effective lagrangian (51) is invariant against the variation of the velocity parameter $V$ under the infinitesimal transformation (44) with the renormalized small component field.

\section{CONCLUSION}

The RPI is an important theoretical issue in the heavy quark effective theory and the NRQCD effective theory. It is required by the consistence of the effective theory. It also leads to interesting applications [10] 11] [12]. The transformation of heavy quark field under the variation of the velocity parameter $V$ proposed by Chen [5] with tree level expression of the small-component field keeps the tree level effective theory invariant. However, at loop level, the transformation needs to be renormalized in the renormalized effective theory. In this paper, I show that the renormalized transformation of the heavy quark keeps the same form as Chen's transform while the small component field needs to be renormalized. I derive the matching conditions for determining the renormalized transformation by imposing an infinitesimal transformation on the relations between the Green's functions in the full QCD and those in the effective theory. These matching conditions are essential for studying the renormalization issue in RPI. As an application of these matching conditions, I determine the renormalized transformation up to order $1 / \mathrm{m}^{2}$. I also show that the previous result in [7] can be understood clearly by building the effective theory in an alternative way, in which the a renormalized effective lagrangian in Dirac four-component field is constructed first, followed by its reduction to the two-component effective lagrangian. The renormalized small component field is then obtained by the equation of motion. The four-component effective lagrangian automatically satisfies RPI. Thus RPI cannot give any constraints on any operators in it. When it is reduced to the two-component effective theory, the same operator with certain coefficient may appear in different terms. The RPI can be used to connect those terms. I also show that the obtained renormalized small component fields by these two methods turn out to be equivalent while the matching conditions provide a 
systematic way to determine the renormalized transformation to any desired order in $1 / \mathrm{m}$ and $\alpha_{s}$ expansions..

\section{APPENDIX A: GENERATING FUNCTIONAL OF GREEN'S FUNCTIONS}

In this appendix, we derive the relations between the Green's functions in QCD full theory and that in the effective theory using generating functional method. It is similar with that given in [13] and [5]. We use the background field method[14, 15] for gluon field interactions, which preserves explicitly the gauge covariant.

In QCD full theory, the generating functional reads

$$
Z[\eta, \bar{\eta}, J, B]=\int \delta[\eta, \bar{\eta}, A] \exp i \int d^{4} x\left(I_{Q}(x)+I_{g}(x)\right)
$$

where $\eta, \bar{\eta}, J$ are the external sources for heavy quark, antiquark and gluon field, $B$ is the background gluon field, $I_{g}$ is given by

$$
I_{g}(x)=-\frac{1}{4} F_{\mu \nu}^{a} F^{a \mu \nu}-\frac{1}{2 \xi}\left(G^{a}\right)^{2}+\ln \operatorname{det}\left[\frac{\delta G^{a}}{\delta \omega^{b}}\right]+J_{\mu}^{a} A^{a \mu}
$$

with

$$
\begin{gathered}
F_{\mu \nu}^{a}=\partial_{\mu}(A+B)_{\nu}^{a}-\partial_{\nu}(A+B)_{\mu}^{a}+g f^{a b c}(A+B)_{\mu}^{b}(A+B)_{\nu}^{c}, \\
G^{a}=\partial_{\mu} A_{\mu}^{a}+g f^{a b c} B_{\mu}^{b} A^{c \mu},
\end{gathered}
$$

being the gauge-fixing term. If $J_{\mu}$ satisfies the following relation

$$
\frac{\delta W}{\delta B_{\mu}^{a}}+\int d^{4} y\left[\frac{\delta W}{\delta J_{\nu}^{b}} \frac{\delta J_{\nu}^{b}(y)}{\delta B_{\mu}^{a}}\right]=-J_{\mu}^{a},
$$

with $W[\eta, \bar{\eta}, J, B]=-i \ln Z[\eta, \bar{\eta}, J, B], W[\eta, \bar{\eta}, J, B]$ is just the effective action regarding to the gluon field $B$ with gauge-fixing term

$$
G^{a}=\partial_{\nu}(A-B)_{\nu}^{a}+g f^{a b c} B_{\mu}^{b} A_{\nu}^{c}
$$

and $I_{Q}$ reads

$$
I_{Q}(x)=\bar{\Psi}(x)(i \not D-m) \Psi(x)+\bar{\eta}(x) \Psi(x)+\bar{\Psi}(x) \eta(x),
$$


The quark field can be integrated out formally and then we have

$$
Z[\eta, \bar{\eta}, J, B]=\int \delta[A] \operatorname{det}[i \not D-m] \exp i \int d^{4} x\left(I_{Q}^{\prime}+I_{g}\right)
$$

where $I_{Q}^{\prime}$ remains the same form as $I_{Q}$. But the quark field now is related to the external source $\eta(x)$ by the following equation of motions:

$$
(i \not D-m) \Psi(x)=-\eta(x)
$$

The generating functional of the effective theory is similar with that of the full theory except the heavy quark action. The effective lagrangian is substituted by (46). In the external source term of the heavy quark only the large component effective field defined in (11) couples to the external source. The action of the heavy quark is given by:

$$
I_{Q}^{V+}(x)=\bar{\Psi}(x) O(x) \Psi(x)+\bar{\eta}(x) \mathrm{P}_{+} h_{V+}(x)+\bar{h}_{V+}(x) \mathrm{P}_{+} \eta(x)
$$

Similarly, integrating out the heavy quark field, the generating functional takes the same form as (A8) with the effective action of the heavy section is substituted by

$$
I_{Q}^{\prime V+}(x)=\bar{h}_{V}(x) \bar{O}(x) h_{V}(x)+\bar{\eta}(x) \mathrm{P}_{+} h_{V+}(x)+\bar{h}_{V+}(x) \mathrm{P}_{+} \eta(x),
$$

with $h_{V}(x)=h_{V+}(x)+h_{V-}(x)$.

The quark field now is related to the external source $\eta(x)$ by the following equation of motions:

$$
\bar{O}(x) h_{V+}(x)=-\mathrm{P}_{+} \eta(x) .
$$

Multiplying $\mathrm{P}_{-}$on both sides, the right hand vanishes and we obtain the renormalized equation of motion:

$$
\mathrm{P}_{-} \bar{O}(x)\left(h_{V+}(x)+h_{V-}(x)\right)=0
$$

This is just the equation (48). The renormalized $h_{V-}(x)$ can be related to $h_{V+}(x)$ by (49). With the equation of motion (A13), (A11) can be simplified as

$$
I_{Q}^{\prime V}=\bar{h}_{V+}(x) \bar{O}(x) h_{V}(x)+\bar{\eta}(x) \mathrm{P}_{+} h_{V+}(x)+\bar{h}_{V+}(x) \mathrm{P}_{+} \eta(x) .
$$

This gives the effective lagrangian density (51). 
The quark determinant in (A8) is responsible for the contributions of the heavy quark loop. It is the same in the full theory and in the effective theory and is suppressed by $1 / \mathrm{m}^{2}$ at least. Thus we may ignore it.

The full quark propagator with background field $B^{\mu}(x)$ is gained by differentiating over the external sources.

$$
G(x, y ; B)=\frac{\delta^{2}}{\delta \eta(x) \bar{\delta} \eta(y)} W(\eta, \bar{\eta}, J, B) .
$$

If the hard cutoff energy scale is set to $\Lambda_{f}$, the same with that in the QCD full theory, the $O(x)$ is then to be $i D-m$, just the same with that in the full theory. The effective lagrangian is just the nonlocal form (3). In this case the only difference of the effective theory and the full theory is the external source term. One immediately gains the relations between the Green's functions of the full theory and those in the effective theory (8). This relation ensures that the nonlocal effective theory is equivalent to the QCD full theory. The local effective theory with the hard cutoff regularization scale $\Lambda_{e}$ is equivalent to that the nonlocal effective theory with hard cutoff $\Lambda_{f}$. This ensures the validation of the relation (8).

[1] M. A. Shifman and M. B. Voloshin, Sov. J. Nucl. Phys. 45, 292 (1987) [Yad. Fiz. 45, 463 (1987)]; M. A. Shifman and M. B. Voloshin, Sov. J. Nucl. Phys. 47, 511 (1988) [Yad. Fiz. 47, 801 (1988)]; N. Isgur and M. B. Wise, Phys. Lett. B 232, 113 (1989); Phys. Lett. B 237, 527 (1990); H. D. Politzer and M. B. Wise, Phys. Lett. 206B, 681 (1988); Phys. Lett. 208B, 504 (1988). E. Eichten and B. Hill, Phys. Lett. B 234, 511 (1990); Phys. Lett. B 240, 447 (1990); B. Grinstein, Nucl. Phys. B 339, 253 (1990); A. F. Falk, H. Georgi, B. Grinstein and M. B. Wise, Nucl. Phys. B 343, 1 (1990); H. Georgi and M. B. Wise, Phys. Lett. B 243, 279 (1990); M. Neubert, Phys. Rept. 245, 259 (1994).

[2] W. E. Caswell and G. P. Lepage, Phys. Lett. B 167, 437 (1986). G. P. Lepage and B. A. Thacker, Nucl. Phys. Proc. Suppl. B4, 199 (1988);

G. T. Bodwin, E. Braaten and G. P. Lepage, Phys. Rev. D 51, 1125 (1995) [Erratum-ibid. D 55, 1125 (1995)].

[3] M. E. Luke and A. V. Manohar, Phys. Lett. B 286, 348 (1992).

[4] A. V. Manohar, Phys. Rev. D 56, 230 (1997) arXiv:hep-ph/9701294.

[5] Y. Q. Chen, Phys. Lett. B 317, 421 (1993). 
[6] M. Finkemeier, H. Georgi and M. McIrvin, Phys. Rev. D 55, 6933 (1997).

[7] W. Kilian and T. Ohl, Phys. Rev. D 50, 4649 (1994) arXiv:hep-ph/9404305.

[8] R. Sundrum, Phys. Rev. D 57, 331 (1998).

[9] C. Balzereit, hep-ph/9809226 (unpublished).

[10] M. Neubert, Phys. Lett. B 306, 357 (1993).

[11] Y. Q. Chen and Y. P. Kuang, Z. Phys. C 67, 627 (1995).

[12] Y. Q. Chen, Y. P. Kuang and R. J. Oakes, Phys. Rev. D 52, 264 (1995).

[13] T. Mannel, W. Roberts and Z. Ryzak, Nucl. Phys. B 368, 204 (1992).

[14] B. S. Dewitt, Phys. Rev. 162, 1195 (1967).

[15] L. F. Abbott, Nucl. Phys. B 185, 189 (1981). 